cette espèce n’a pas été indiquée sur ces figures, pour ne pas les compliquer.

La figure 1 représente une antenne normale de Pyrrhocoris apterus, et les huit autres des antennes anomales de cette espèce, que j'ai choisies parmi les plus intéressantes. Toutes les autres antennes anomales de l'espèce en question examinées par moi étaient semblables ou analogues à celles figurées dans cette note.

Les figures 1-6 et 9 représentent des antennes gauches, et les figures 7 et 8 des antennes droites.

Je suis très porté à croire que ces anomalies antennaires sont des anomalies de régénération; mais ce n'est qu'une hypothèse qui a besoin de confirmations expérimentales.

Comme il s'agit d'une simple note, je ne crois pas devoir indiquer les travaux que je connais sur cette question de tératologie entomologique.

\title{
L'appareil copulateur de Tenthredella temula Scop. [HrM.]
}

\section{par H. Boulangé.}

On a déja beaucoup écrit sur l'appareil génital màle des Insectes, tant sur les organes internes que sur l'appareil copulateur. Mais, pour celui-ci, le plus souvent, une description des pièces externes a seule été donnée. Pour les Hyménoptères, les travaux portent presque toujours sur les Aculéates (1).

(1) Nous ne pouvons donner ici la bibliographie complète de notre sujet; elle serait hors de proportion avec cette simple note. On pourra se rendre un compte exact du point où nous trouvons la question.

$1^{\circ}$ par les traités généraux sur les Insectes :

190i. Hennegur : les Insectes, - ne cite pas de Chalastogastres.

1909. Berlese : gli Insetti, vol. I, - ne décrit que Cimbex, brièvement.

1912. Schroder : Handbuch der Entomologie - l'apfareil copulateur n'est pas encore paru.

1914. Escherich : die Forstinsekten Mitteleuropas, Bd I, - ce traité ne comporte pas l'étude détaillée de la question.

$2^{\circ}$ par les principaux mémoires originaux :

1884 à 93. Radoszkowsky : diverses études sur les armures d'Aculéates en vue de la détermination. 
C'est ce qui nous a poussé à entreprendre l'étude de l'appareil génital des Chalastogastres.

On a souvent discuté sur la valeur spécifique de l'appareil copulateur tour a tour exaltée et niée. Cela provient sans doute de ce que cette spécificité n'est pas la mème dans tous les genres, mais aussi de ce qu'on n'a peut-ètre pas assez sérié les caractères faute de distinguer entre les pièces dont la forme est liée a l'acte de la copulation et celles dont la forme est plus indifférente.

Or la physiologie de chaque partie est difficile à observer, surtout pour les insectes rarement rencontrés in copula et chez qui l'acte est bref. C'est alors la musculature de l'appareil qui nous renseignera le mieux. Malheureusement, la bibliographie se réduit à quelques noms seulement si l'on s'en tient aux mémoires qui l'ont étudiée (1).

La musculature montrera aussi les homologies, conjointement avec le développement qui, lui, a fait l'objet de diverses études $\left({ }^{2}\right)$.

Nous avons déjà disséqué les appareils mảles d'un certain nombre de Chalastogastres récoltés dans le Nord de la France, et, en attendant que nous ayons recueilli assez de documents pour un travail d'ensemble, nous nous proposons de communiquer a la Société entomologique nos principaux résultats.

Parmi les espèces déja disséquées, celle qui nous paraît fournir le

1893, 94, 95. Bordas : divers mémoires ne parlant que des Aculéates et des Ichneumonidae.

1895. Pevtoureau : thèse, - n'étudie pas les Hyménoptères, personnellement. 1900 et 1903. Zinder : deux mémoires sur l'appareil màle des Hyménoptères et sur celui des Insectes, - éludie Cimbex et surtout Sirex.

1908. Severin H. et H. : sur Cimbex americana, - ne decrivent que les organes internes.

1912. Ensuiv : commence la publication des Tenthrédiniens de l'Europe centrale par une brève étude anatomique, - la description de l'appareil génital est faite d'après ZINDER.

Les autres mémoires que nous pourrions citer traitent d'ordres autres que les Hyménoptères ou sont spéciaux à certains Aculéates, comme celui de Kluge (1895) sur Vespa germanica.

(1) Nous pourrions citer :

1895. Kluge: Das mannliche Geschlechtsorgan von Vespa germanica.

1895. Pextocreac : Contribution à l'élude de la morphologie de l'armure génitale des insectes, - thèse.

(2) Le développement de l'appareil copulateur a été étudiẻ par Zander.

Les travaux bien connus de Herold (1815), Tichomiroff (1880), Nusbaum (1882), Verson (1895), Michielis (1900) traitent surtout du développement des organes internes. 
meilleur type morphologigue est Tenthredella temula (Scop .). Certaines espèces ont des pièces chitineuses bien définies se distinguant nettement des membranes souples par leur coloration foncée; chez d'autres, le revêtement chitineux, presque uniforme, ne présente pas ces "pièces ". Ici, nous avons un type intermédiaire qui montre bien ce

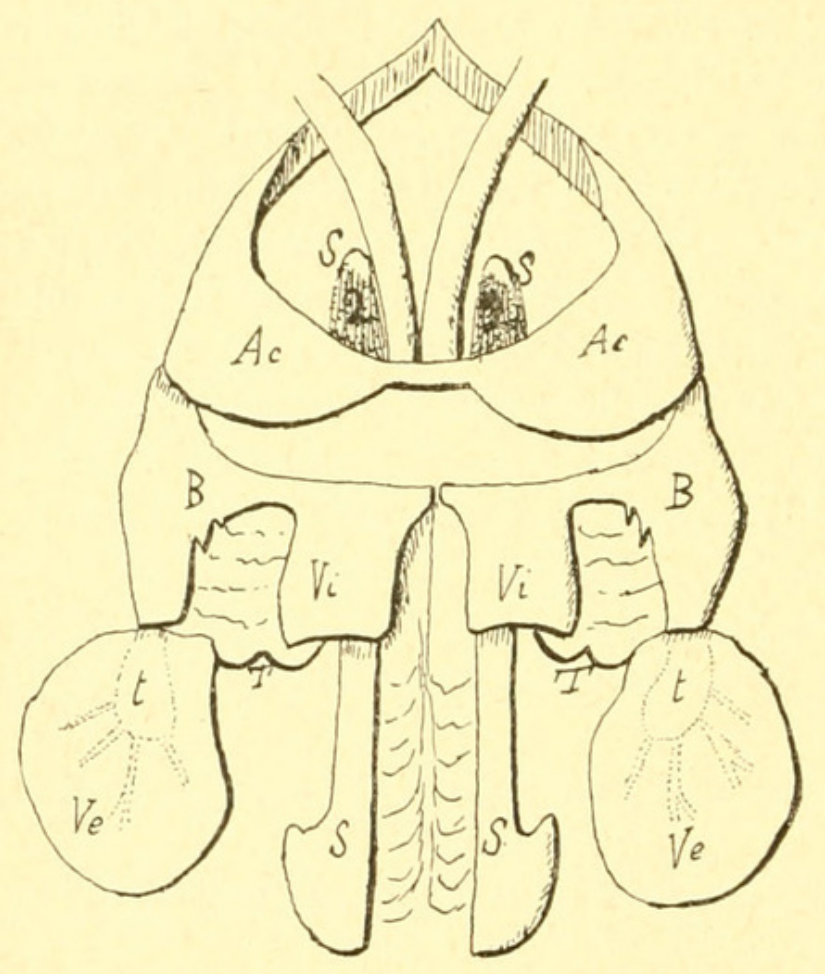

Fig. 1. - Appareil copulateur de Tenthredella temula (face ventrale). qu'exprime cette locution "pièce chitineuse " prise souvent dans un sens trop absolu faisant perdre de vue la continuité du revêtement.

Il n'y a pas, d proprement parler, de parties dures réunies par des articulations; mais il $y$ a partout une membrane chitineuse qui se renforce là où besoin est, peut présenter de grands espaces minces et souples et parfois des surfaces apparaissant en dégradé; on ne sait plus alors assigner de limite précise à la "pièce chitineuse ".

Il y a pourtant des régions constamment épaissies auxquelles on peut appliquer une nomenclature. Nous en avons déjà plusieurs.

Les plus usitées sont dues à Dufour, André, Kluge, Mrchaelis, ZANDER. Elles sont bien établies et fort utiles pour les groupes pour lesquels elles ont été concues. Mais en appliquant aux Chalastogastres une nomenclature bonne pour les Bombus, par exemple, nous nous exposerions à préjuger d'homologies non vérifiées. Nous ne nous servirons donc de termes rappelant ces nomenclatures que là où l'homologie n'est pas douteuse; ailleurs, nous les éviterons, nous contentant de rappeler entre parenthèses les termes déjà employés par les auteurs.

L'appareil copulateur de Tenthredella temula Scop. se compose d'une pièce basilaire (cardo) (B) ayant la forme générale d'un tronc de còne. La base proximale est coupée selon un plan oblique sur l'axe, 
de manière à donner plus de hauteur du còté dorsal. Le bord distal est profondément découpé de la manière suivante :

10 sur les côtés, la pièce basilaire est longue;

2 sur la face ventrale deux échancrures symétriques et une médiane isolent deux lobes lames internes, hinnere Haltezáng 2 de Zander et Evslin) (Vi.);

$3^{\circ}$ sur la face dorsale, le tronc de cône est profondément échancré au point d'ètre interrompu sur la ligne médiane. Cette grande échancrure est en partie comblée par une paire de plaques indépendantes (C.)

L'ensemble est couronné à l'extrémité proximale par une pièce annulaire, c'est le « sclerite accessorio di rinforzio » de BerLese (Ac). ZANDER et après lui EnsLin en font le cardo, et ce que nous avons

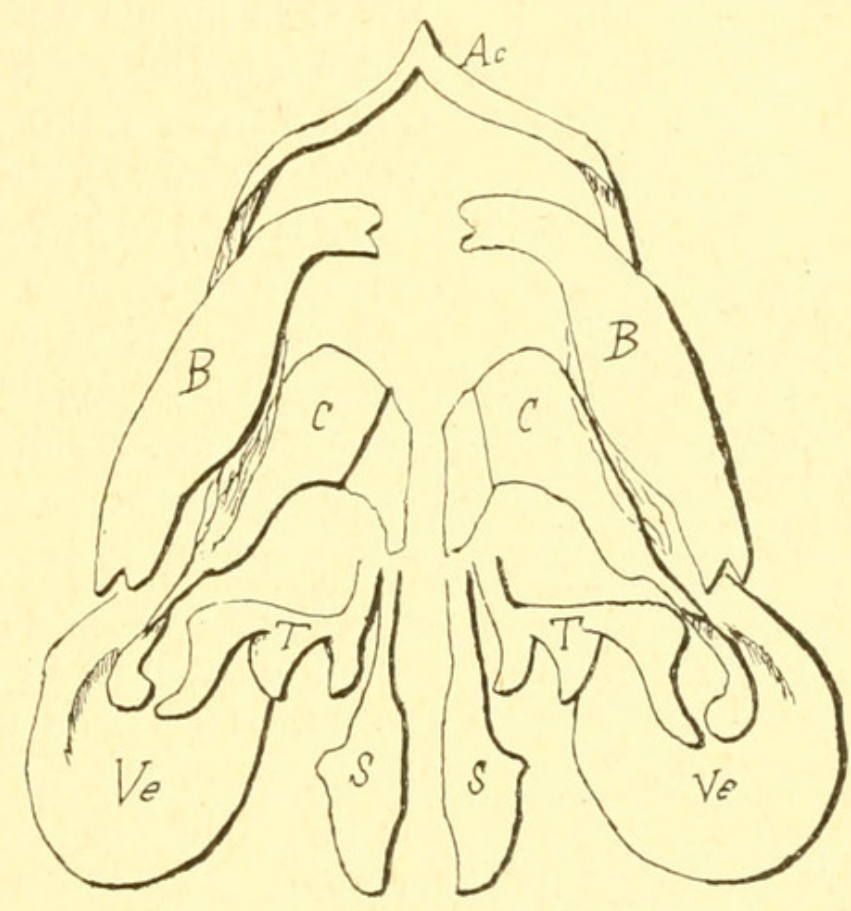

Fig. \%. - Appareil copulateur de Tenthredella temula (face dorsale). appelé pièce basilaire (cardo) est pour eux le tronc commun (gemeinsamen Stamme) des lames externe et internes désigné par St. sur leurs dessins. Cet exemple suffira a justifier notre réserve touchant les nomenclatures. Nous nous rallions plus volontiers au terme de BerLese, car cette pièce' n'est pas constante chez les Chalastogastres.

D'ailleurs, nous n'en parlerons pas davantage dans cette note, parce que cet anneau ne porte pas d'insertions de muscles péniens.

A l'extrémité distale, les flancs de la pièce basilaire se terminent par une palette articulée (lame externe) (Ve).

Du mème còté, les pièces qui comblent l'échancrure dorsale de la pièce basilaire se prolongent par un crochet tordu de 90 degrés. Ce crochet, dont les variations sont fort intéressantes à suivre, ne se voit en saillie sur le reste de l'appareil que chez Sirex, où il est denti. ZANDER et Exslin le représentent sous le nom de hinnere Haltezang 1. plus dorsale, comme de juste que l'autre lame interne. 
Enfin tandis que l'extrémité proximale du tronc de còne est ouverte. la cavité générale du corps étant par là en continuité avec celle de l'appareil copulateur, la base distale est fermée par une membrane chitineuse, comme le reste de l'appareil. Cette membrane présente une série de bosses à la surface desquelles elle s'épaissit; la plus externe de ces bosses forme avec le crochet ter-

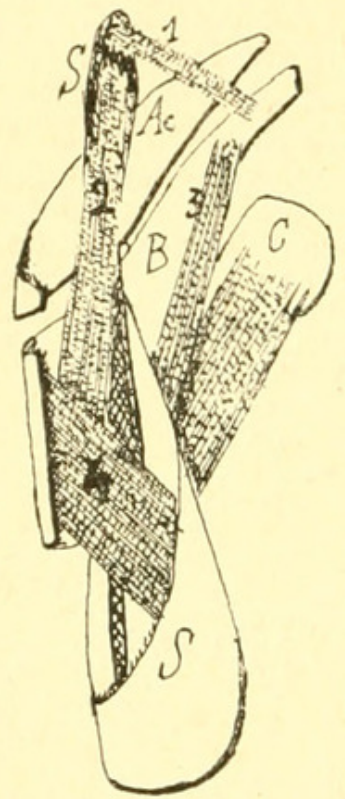

Fig. 3. - Demi-appareil copulateur droit face interne). minant la plaque dorsale, une petite pince. La série des bosses est relativement rigide et l'étude d'autres types lui fait donner la valeur d'une pièce véritabie $(\mathbf{T})$. Son extrémité.interne remonte contre la gaine du pénis et s'applique sur la valve correspondante.

La gaine du pénis (fourreau) est elle-même, comme on le sait, composée de deux valves (spatha) faiblement unies entre elles, de sorte que tout l'appareil copulateur se laisse aisément sectionner selon son plan de symétrie. Pour cela, il suffit de séparer les valves du pénis, de profiter de l'interruption dorsale de la pièce basilaire, et du côté ventral, de suivre au scalpel une petite fente médiane qui, pour ètre moins visible, n’en sépare pas moins le pont étroit qu unit les lames in. ternes.

On peut alors considérer le demi-appareil copulateur vu par sa face d'union avec l'autre moitié (fig. 3 ).

Au premier plan est la spatha $(\mathbf{S})$ où l'on peut distinguer deux parties :

$1^{\circ}$ La partie distale, en saillie; elle a la forme d'une palette à face interne plane et dont la face externe, bombće, porte un petit tubercule. Cette partie est creuse tout comme les autres pièces en saillie que nous avons décrites, ce sont des évaginations, non des apophyses. La cavité communique avec la cavité générale par une fente oblique ventrale.

2. La partie proximale : tige étroite et particulièrement résistante; elle est comme le manche de l'autre. Ce manche prend naissance sur la première partie sous forme d'épaississement de sa paroi externe et se prolonge à travers tout l'appareil copulateur jusqu'à dépasser légèrement (a l'état de rétraction) le niveau du bord proximal dorsal de la pièce basilaire. 
Nous n'entrons pas dans le détail de la structure de la spatha dont il est plus intéressant de faire des études comparatives, nous avons déja rassemblé des matériaux dans ce but, nous nous proposons d'y revenir, ainsi que sur les organes internes.

Musculature. - Les mouvements de saillie et de retrait de la spatha sont produits par quatre muscles :

$1^{\circ}$ Le protracteur dorsal (1) s'insérant sur l'extrémité proximale du manche et sur la partie étroite dorsale de la pièce basilaire.

2 Le protracteur ventral (2) s’insérant aussi sur l'extrémité du manche et d'autre part dans le fond de l'évagination qu'est la lame interne.

$3^{\circ}$ Le rétracteur dorsal ( 3 s'insérant au raccord des deux parties de la spatha et sur la partie étroite dorsale de la pièce basilaire, à côté du protracteur dorsal.

$4^{\circ}$ Le rétracteur ventral (4), aplati, sortant par la fente de la spatha et s'attachant le long de l'échancrure médiane ventrale de la pièce basilaire.

A ces quatre muscles s'en ajoute un autre très grêle $(\breve{)})$, il s'insère sur la spatha a còté du rétracteur dorsal, part

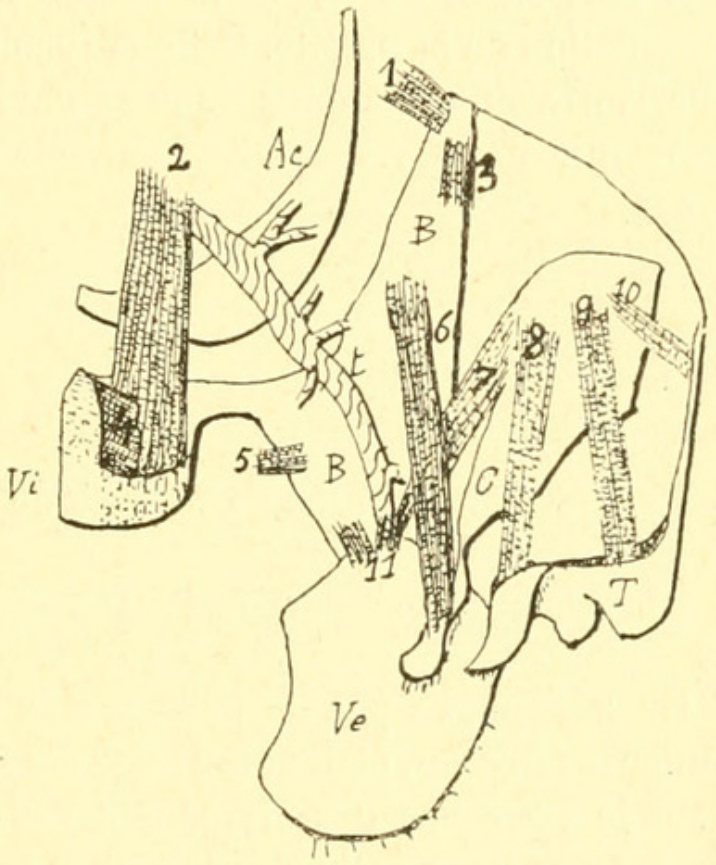

Fig. 4. - Demi-appareil copulateur droit, spatha enlevée, les autres pièces étalées dans un même plan. perpendiculairement à elle et va s'insérer sur la pièce basilaire dars la partie latérale. Ce muscle et son symétrique, en se contractant, écartent donc les spatha l'une de l'autre produisant le mouvement des baguettes a bàtonner les gants. Les deux spatha n'étant pas unies par des muscles susceptibles de provoquer le mouvement antagoniste, nous devrons en chercher les agents ailleurs.

Outre ces muscles s'insérant sur la spatha, il existe encore deux muscles croisés en $\mathrm{x}$ qui doivent produire une oscillation, un va-etvient le long de la pièce basilaire, de la pièce qui la complète du còté dorsal; de ces deux muscles, c'est le protracteur qui est externe (fig. 4-7); c'est lui qui occasionne une boursouflure de la membrane qui est représentée sur la figure 2. L'autre muscle (6) s'insère dans 
le crochet même de la pièce complémentaire dorsale. Cette pièce, à son tour, porte sur sa partie élargie les insertions des muscles se rendant à la pièce bosselée T ; l'un (8) va dans la bosse externe formant pince, un autre (9) dans la bosse interne et quelques fibres (10) à la partie qui remonte le long du pénis. Cette dernière pièce T peut donc exécuter un mouvement de bascule; outre le resserrement de la pince dont nous avouons mal comprendre le rôle là oủ elle n'est pas plus développée que chez Tenthredella, il faut sans doute rechercher ici l'agent susceptible de rapprocher les spatha.

Il faut encore citer deux muscles (11), faibles ici, qui produisent l'oscillation de la palette. Celle-ci reçoit en outre une grosse trachée $(t)$ que l'on voit s'épanouir dans sa cavité (fig. 1) et un nerf important mais que nous reconnaissons avoir remarqué surtout dans d'autres types.

\section{Sur le parasitisme externe des Braconides [Нyм.]}

par F. Picard et É. Rabaud.

On a supposé pendant longtemps que les Braconides, et en particulier les Bracon, étaient tous des parasites internes, et T. A. Marshall, dans le "Species des Hyménoptères d'Europe " admet encore, en se basant sur l'anatomie des larves et la disposition de l'appareil respiratoire, que toutes les larves de Braconides doivent vivre dans l'intérieur du corps de leur hôte. Cependant, depuis les observations très anciennes de Brischke et de Ratzeburg, et celles plus modernes de SEurat et d'autres auteurs, il est prouvé que le parasitisme externe est fréquent, surtout chez les espèces dont les victimes sont lignivores et enfermées dans une galerie ou une cavité close.

Presque toutes les espèces du genre Bracon se développent aux dépens de larves vivant dans les tissus des végétaux, Cynipides, Curculionides, Scolytides, Anobiides, Tortricides, Sésies, Muscides, etc. Mais leur biologie n'a généralement donné lieu à aucune étude précise, et on s'est presque toujours contenté de signaler les adultes éclos dans les élevages. Il semble bien, toutefois, que le parasitisme externe est la règle chez les Bracon; il en est du moins ainsi pour les Bracon, notamment B. variator Nees, parasites des larves vivant dans les capitules des Carduacées (Larimus, Myelois cribrella), et pour le Microbracon 


\section{$2 \mathrm{BHL}$ Biodiversity Heritage Library}

Boulangé, H. 1914. "L'appareil copulateur de Tenthredella temula Scop. (Hym.)." Bulletin de la Société entomologique de France 1914, 260-266.

View This Item Online: https://www.biodiversitylibrary.org/item/34151

Permalink: https://www.biodiversitylibrary.org/partpdf/76350

\section{Holding Institution}

Smithsonian Libraries

\section{Sponsored by}

Smithsonian

\section{Copyright \& Reuse}

Copyright Status: NOT_IN_COPYRIGHT

This document was created from content at the Biodiversity Heritage Library, the world's largest open access digital library for biodiversity literature and archives. Visit BHL at https://www.biodiversitylibrary.org. 